\section{Event Streamed Spectrum Imaging using Programmed Beam Acquisition in Biological Microprobe Analysis}

Peter Ingram, ${ }^{\star}$ Scott D. Davilla, ${ }^{\star *}$ and Ann LeFurgey

${ }^{*}$ Duke University and Veterans Affairs Medical Centers, Durham, NC ${ }^{*} 4$ pi Analysis Inc., Durham, North Carolina, NC p.ingram@cellbio.duke.edu

\section{Background}

The general trend of microscopical investigation in biology from the 1950s to the early 1970s was towards obtaining structural information. This goal initially was met using heavy metal and/ or aldehyde fixatives, room temperature dehydration with polar organic liquids, embedding with epoxy and acrylate resins, and thin sectioning at room temperature. By the mid 1970s, a perceptible change occurred in the direction of both light and analytical electron microscopy towards investigation of the chemical reactivity and composition of structures made visible with increasingly better spatial resolution for light and electron microscopes. During the past 25 years there have been considerable innovations in microanalytical techniques, including analytical electron and $\mathrm{x}$-ray microscopy and microanalysis, secondary ion mass spectrometry, laser microprobe mass analysis, the scanning probe microscopies, and confocal/multi-photon microscopy. In addition, cryopreservation and the development of chromophores for visualization of molecular and ionic sites within individual living cells as well as membranes have redefined the goal of microscopical preservation: to stabilize cell structure and composition as they exist in the living state.

\section{Analytical imaging}

In biology, this term is often used in the context of acquisition and analysis of compositional data at cellular and subcellular spatial resolutions using an electron microscope. Clearly, this is only one aspect of a very broad field of scientific inquiry that generally falls under the rubric of "Compositional Imaging" and can involve many different sources of radiation including electrons, ions, and photons. Subtle distinctions in biological microscopy exist between "structural microanatomy" and "chemical microanatomy"; they obviously can be significantly different, for example during the early stages of cell injury when changes in the elemental distribution within a cell can become readily apparent before any observable changes in morphology via "conventional" transmission electron microscopy.

In addition, it is important to recognize that in general there are only 2 possible ways to form an image: either each picture point is formed simultaneously or each is formed sequentially. In either scenario, quantitative information is retrievable; however, from a practical point of view it is much simpler to perform this on a pixelby-pixel basis. [Both electron energy-loss spectroscopy (EELS) and $\mathrm{x}$-ray spectroscopy in the form of either energy or wavelength dispersive spectroscopies (EDX/WDX) are routinely performed on biological specimens in the (scanning) electron microscope.]

The current trend of $\mathrm{x}$-ray mapping in Electron Probe X-ray Microanalysis (EPXMA) is toward full spectrum collection, or spectrum imaging [1]. Spectrum imaging is the collection and spatial registration of all $\mathrm{x}$-ray events, yielding a spectral data cube. Recent developments in spectral analytical techniques have greatly improved the efficiency and precision of data handling from quantitative chemical images. Principal component and cluster analyses have significantly aided in unambiguous identification and assessment of localized data as well as the reduction of statistical noise in the images. Fitting of all elemental peaks at each pixel to standards accomplishes a similar result as well as providing real-time or off-line quantitative images. "Programmed beam acquisition" (PBA) enables the exciting beam to only dwell for sufficient times per pixel in regions of the specimen necessary to acquire a predetermined statistical precision (e.g. \pm 2 standard deviations). Curiously PBA, while not a new concept [1,2], is rarely used. However, it is of particular importance to biological samples, which are sensitive to beam damage and inherently produce low

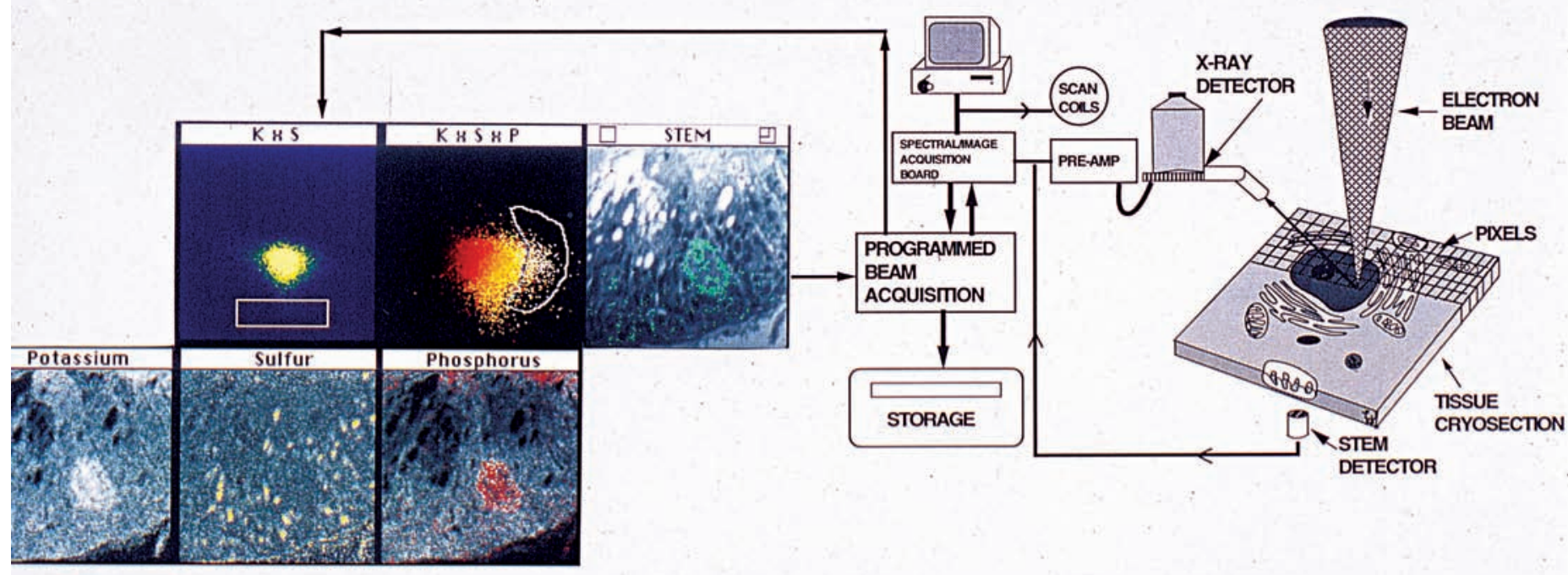

\title{
$\mathbf{5} \mu \mathrm{m}$
}

\section{INTERACTIVE QUANTITATIVE EPXMA}

Fig.1. Slow scan conventional Programmed Beam Acquisition. Regions selected from scatterplots between various elemental maps (Potassium, Phosphorus and Sulfur from freeze-dried cryosections of mouse kidney) are used to generate masks that are assigned different pixel dwell times for acquisition of $x$-ray data [1]. Reproduced with author's permission. 


\section{Let 4pi take you beyond the capability of any SEMISTEM on the market with}

RevolutionEDX

X-ray Microanalysis Systems

Save time and production costs with 4pi's unique features

Features available exclusively from 4pi:

Event-Streamed Spectrum Imaging ${ }^{\text {m }}$

and

Spatial Frame Lock $^{\text {TM }}$

Real-time electron-beam drift correction
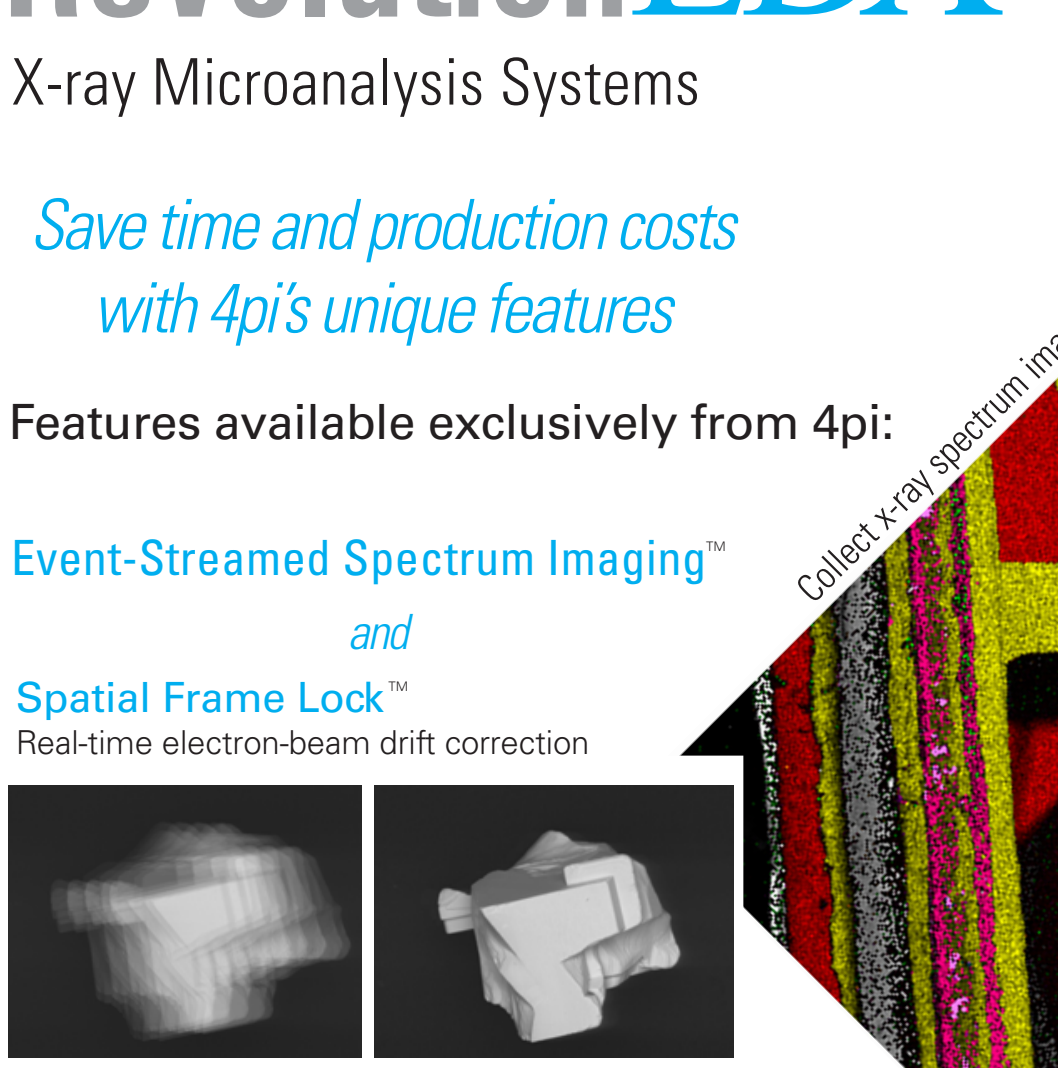

X 


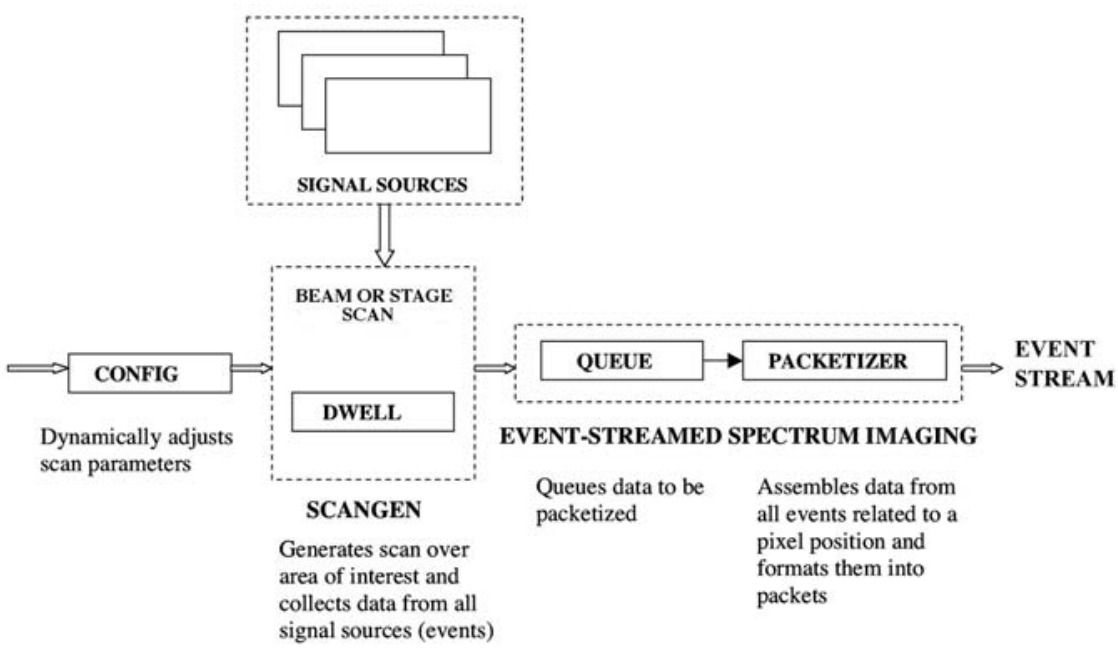

Event Stream Spectrum Imaging Schematic

Fig.2. Event Stream Spectral Imaging (ESSI) schematic

$\mathrm{x}$-ray count rates. Using traditional collection methods, long collection times can be required [1] in order to reach the statistics necessary for accurate quantitative data acquisition. PBA can significantly reduce this time by using one or more image masks to define areas of varying $\mathrm{x}$-ray dwell times (Fig. 1). Optimal dwell time is spent over areas of interest while minimal time is spent over sample support substrates.

Event Streamed Spectrum Imaging (ESSI) is a new method [3, 4] that overcomes many of the limitations in conventional $\mathrm{x}$-ray mapping acquisition alluded to above. X-ray events are treated as simply another signal source (Fig. 2), requiring scan generation hardware that is closely coupled to the $\mathrm{x}$-ray pulse processor. While

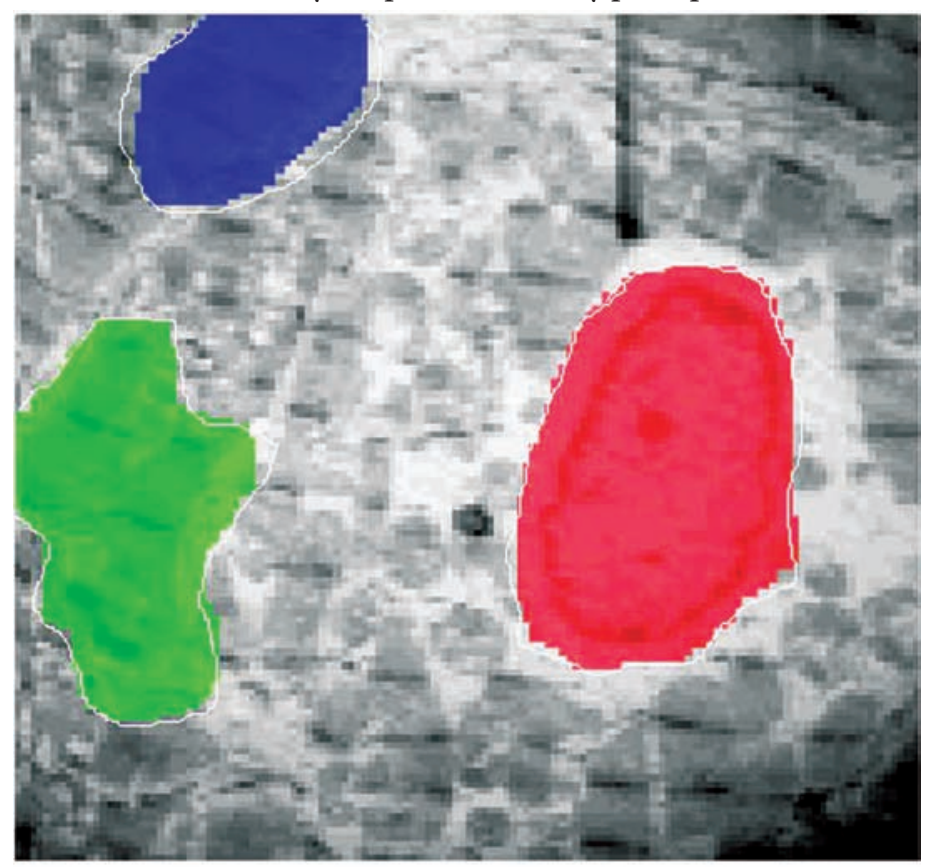

Fig.3. Dynamic Dwell Modulation. Chemically fixed, stained and embedded thin section of frog cardiac muscle. Masks are drawn around regions of the cells in which a dwell times (clockwise from top left) of 16, 40 and $80 \mathrm{msec} /$ pixel were applied. A dwell time of $8 \mu \mathrm{sec} /$ pixel was applied to the rest of the field. The image was frame locked to prevent drift. (Dark area at top right is beam damage from an earlier scan that is common in Epon embedded sections.) this coupling has become standard with EDX systems offering spectrum imaging, stream-based acquisition differs in the treatment of the collected data. Instead of passing the current $x-y$ position to the X-ray pulse processor for registration, X-ray events are passed to the scan generator and assembled into a pixel event that contains all information related to that pixel position. The pixel events are streamed as packets to a host computer, where they are buffered and stored to disk for analysis by an independent process. A high degree of interactive analysis is possible, including real-time alteration of acquisition conditions such as dynamic dwell modulation (i.e. programmed beam acquisition) where the X-ray dwell is altered on a frame basis to increase (or decrease) dwell times over areas of interest (Fig. 3). Similarly, real-time drift correction can be implemented readily (Fig. 4).

ESSI is also applicable to stage mapping in X-ray Probe X-ray Microanalysis (XPXMA) where the specimen stage is generally rastered across a stationary beam. Instead of the traditional method of stepping the stage to discrete static positions and collecting X-rays, the stage is slewed across the beam position and events that occur are registered to the dynamic stage position. This method eliminates the overhead required in discrete stepping and results in faster stage mapping.

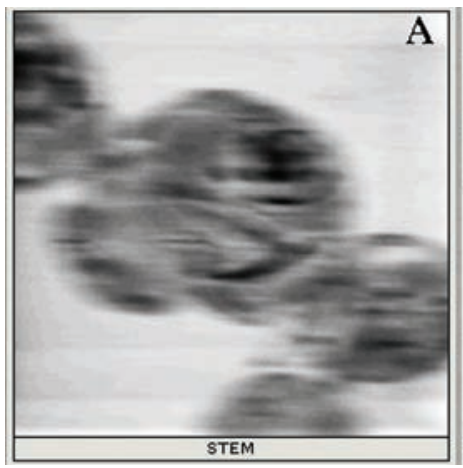

A

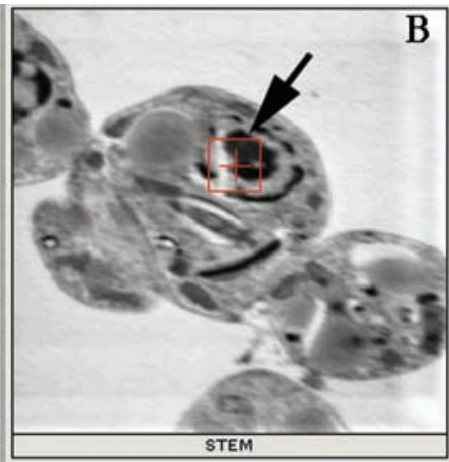

Fig.4. Chemically fixed and stained Leishmania donovani amastigotes (A) without drift correction, (B) with drift correction. Arrow indicates the region of frame lock.

ESSI is an extremely efficient process for the collection of all electron and X-ray information registered to every $x-y$ beam position over single or multiple frames with zero overhead, and allows the collection of X-ray and other events to be truly concurrent with the collection of electron events.

\section{References}

[1] P. Ingram et al. Biomedical Applications of Microprobe Analysis, Academic Press (1999)

[2] P. Ingram, D.A. Kopf, and A. LeFurgey. Microbeam Analysis, J. Friel, Ed., (1994) 111

[3] S.D. Davilla, Scanning 28, 2 (2006) 120

[4] S.D. Davilla, Microscopy \& Microanalysis, 1344CD, (2007)

[5] Most of these studies have been published previously as abstracts at MSA meetings.

- A.L. gratefully acknowledges the support of a US Veterans Affairs Merit Award. 
